\title{
Prompt energy calibration at RENO
}

\section{Sang Yong KIM ${ }^{1}$ on behalf of the RENO Collaboration}

Seoul National University

23-315, Department of Physics \& Astronomy, Seoul National University,

1 Gwanak-ro, Gwanak-gu, Seoul, 08826, Korea.

E-mail: sfc5302@gmail.com

RENO (Reactor Experiment for Neutrino Oscillation) has obtained the first measured value of effective neutrino mass difference from a spectral analysis of reactor neutrino disappearance. The measurement absolutely relies on the accurate energy calibration. Several radioactive sources such as ${ }^{137} \mathrm{Cs},{ }^{54} \mathrm{Mn},{ }^{68} \mathrm{Ge},{ }^{65} \mathrm{Zn},{ }^{60} \mathrm{Co}$, Po-Be, Am-Be, and $\mathrm{Cf}-\mathrm{Ni}$, are used for the energy calibration of the RENO detectors. We obtained an energy conversion function from observed charges to prompt signal energy which describes a non-linear response due to the quenching effect in liquid scintillator and Cherenkov radiation. We have verified the performance of the energy calibration using copious beta decay events from radioactive isotopes B12 that are produced by cosmic-muon interaction. The energy calibration was performed for the target and gamma-catcher regions separately due to their different energy responses. In this presentation we describe the methods and results of the energy calibration.

38th International Conference on High Energy Physics

3-10 August 2016

Chicago, USA

\footnotetext{
${ }^{1}$ Speaker

(C) Copyright owned by the author(s) under the terms of the Creative Commons 


\section{Introduction}

The reactor is detected via the inverse beta decay(IBD) reaction. A pair of a prompt positron signal and a delayed signal from neutron capture by Gadolinium $(\mathrm{Gd})$ or Hydrogen $(\mathrm{H})$. Neutron capture event by $\mathrm{Gd}$ emits $\sim 7.96 \mathrm{MeV}$ gamma rays. And neutron capture by $\mathrm{H}$ emits $\sim 2.2 \mathrm{MeV}$ gamma ray. A prompt signal comes from positron annihilation including positron kinetic energy. So prompt signal emits gamma rays with broad energy range from $1 \mathrm{MeV}$ to $12 \mathrm{MeV}$. To reconstruct right energy from signal, we need charge to energy conversion functions for each detectors. A event energy is determined from the total charge(p.e) which is collected by PMTs. For reference energy points, we use 8 radioactive sources, ${ }^{137} \mathrm{Cs},{ }^{54} \mathrm{Mn},{ }^{68} \mathrm{Ge},{ }^{65} \mathrm{Zn},{ }^{60} \mathrm{Co}$, Po-Be, Am$\mathrm{Be}$, and $\mathrm{Cf}-\mathrm{Ni}$.

\section{Calibration work}

There are two main regions, target and gamma-catcher( catcher), inside our detector. To deploy at the center of target and catcher, we installed mechanical calibration systems which is a step motorized pulley system in a globe box on the detector cover. Radioactive source which is encapsulated by acrylic case could be deployed by rotating pulley with servo motor.

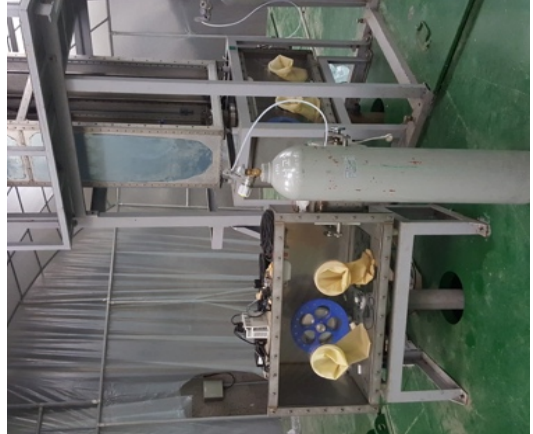

(a)

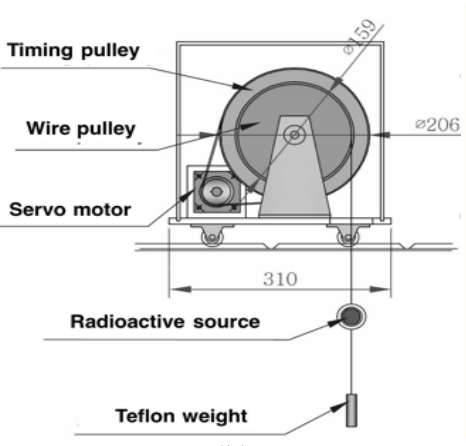

(b)

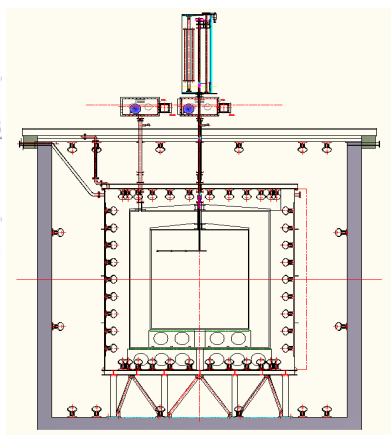

(c)

Figure 1. RENO Mechanical source driving system (a) Pulley schematic, (b) A picture of glove boxes, (c) Calibration system schematic

\subsection{Calibration source charge distribution}

We could have reference charge points which are corresponding specific energy(MeV) from the peak point of charge distribution of each calibration sources. ${ }^{137} \mathrm{Cs}$ radioactive source emits single gamma ray for $0.662 \mathrm{MeV},{ }^{54} \mathrm{Mn}$ emits single gamma ray for $0.835 \mathrm{MeV},{ }^{68} \mathrm{Ge}$ emits two gamma rays for $1.022 \mathrm{MeV}$ as a positron source, ${ }^{65} \mathrm{Zn}$ emits single gamma ray for $1.1 \mathrm{MeV},{ }^{60} \mathrm{Co}$ emits two gamma rays for $2.5 \mathrm{MeV}$, Po-Be \& Am-Be emits single gamma ray for $4.95 \mathrm{MeV}$ as neutron capture by Cabon, ${ }^{252} \mathrm{Cf} \&$ IBD events emit single gamma ray for $2.2 \mathrm{MeV}$ as neutron capture by Hydrogen and emits multiple gamma rays for $7.96 \mathrm{MeV}$ as neutron capture by Gadolinium, and Cf-Ni source emits single gamma ray for $8.9 \mathrm{MeV}$.

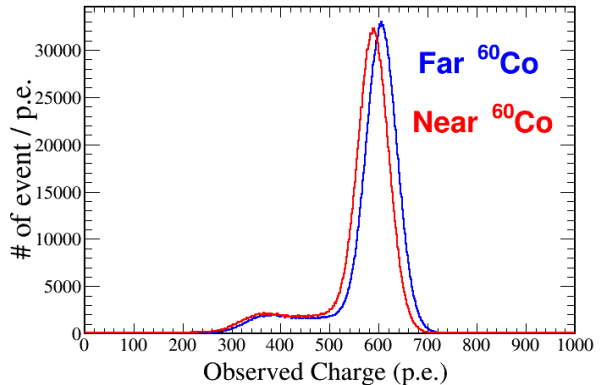

(a)

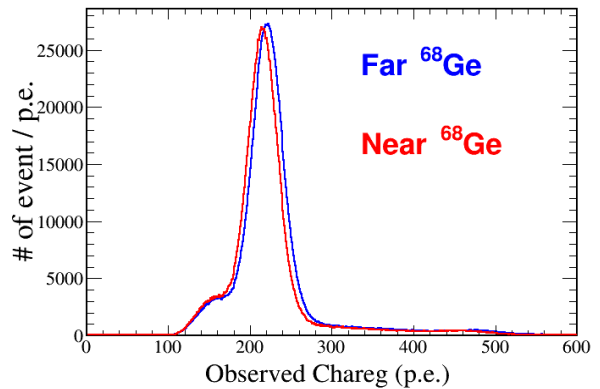

(b)

Figure 2. Calibration sources charge distribution (blue line is far detector, red line is near detector) (a) ${ }^{68} \mathrm{Ge}$ source charge distribution, (b) ${ }^{60} \mathrm{Co}$ source charge distribution. 


\subsection{Correction from multiple gamma to single gamma by MC.}

There are non-linear response of scintillating energy in our liquid scintillator. The quenching effect could be calculated by Monte-Carlo(MC) simulation. For example, ${ }^{60} \mathrm{Co}$ emits two gamma rays for $1.1732 \& 1.3325 \mathrm{MeV}$. First step is generating two gamma rays like as ${ }^{60} \mathrm{Co}$ source. Second is generating single gamma ray for $2.5057 \mathrm{MeV}$. Finally, by comparing both of them, we could have correction factor for ${ }^{60} \mathrm{Co}$ source. By MC results, we could correct quenching effect.

\subsection{Correction from gamma to positron by MC.}

Reactor neutrino prompt signal comes from positron annihilation. So we should correct from gamma ray response to positron. As above method, first step is generating positron by MC. Second is generating gamma ray by MC. By comparing the results of two steps, we could have correction factor for conversion to positron.

\section{Energy conversion function}

We are adapted fitting function as . We could draw p.e / MeV plot for prompt energy. The fitting results are well described the non-linear response of scintillating energy for the corresponding IBD prompt signal. We could also calculate conversion function for electron by subtracting positron annihilation effect. We have verified the performance energy calibration using electron conversion function. We have good results of electron energy spectra of beta decays from radioactive isotope ${ }^{12} \mathrm{~B}$ and ${ }^{12} \mathrm{~N}$, which are produced by cosmic-muon interactions. Electron scintillating energy spectrum have good agreement between data and MC simulation spectrum.

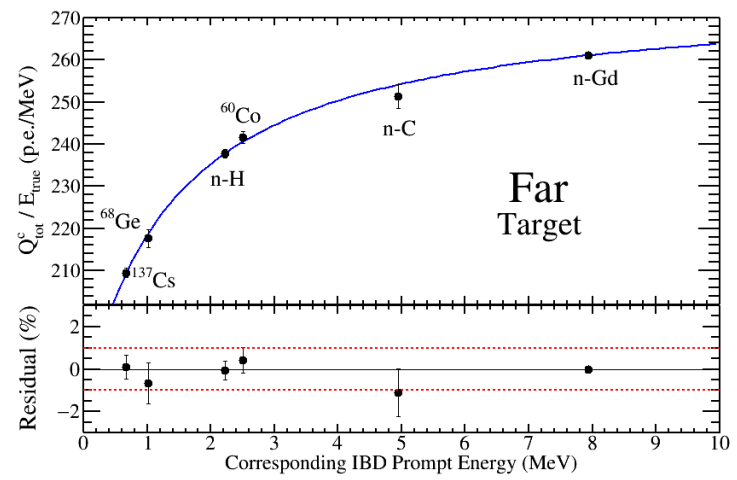

(a)

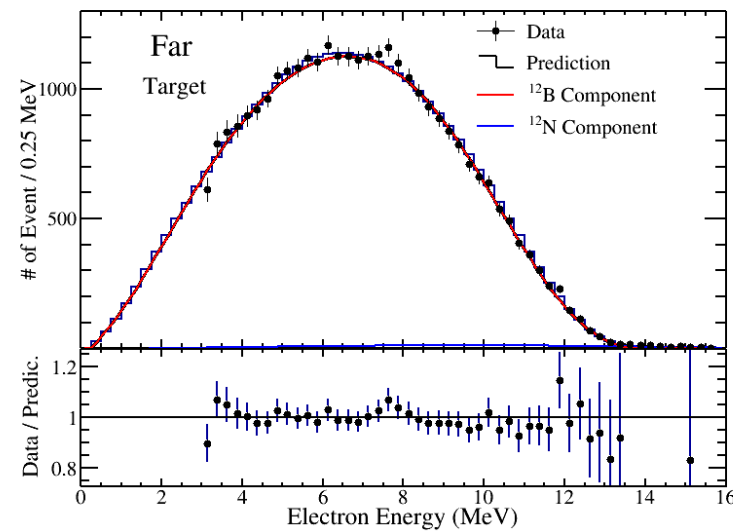

(c)

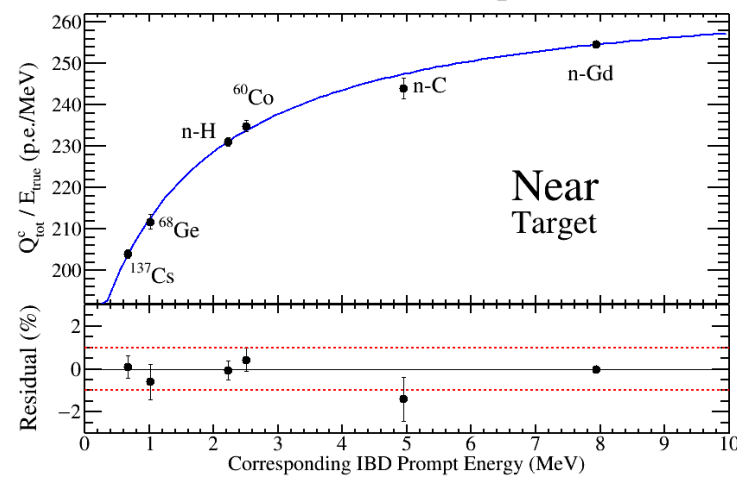

(b)

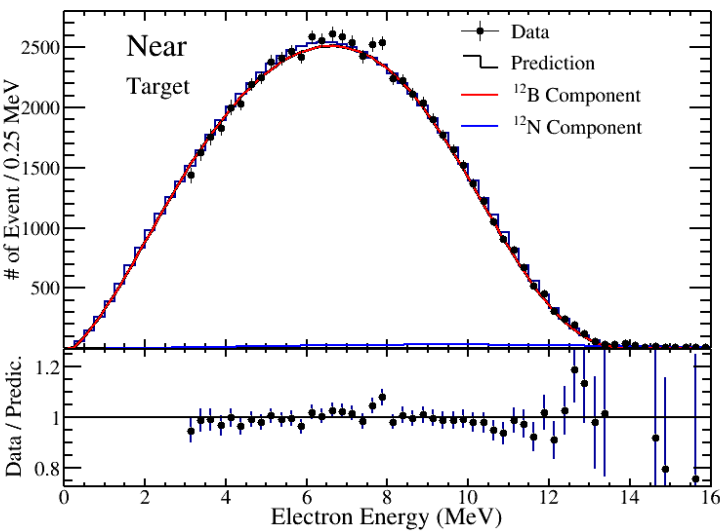

(d)

Figure 3. Charge to energy conversion function fitting result for each detectors

(a) Fit result of far target for IBD prompt energy (b) Fit result of near target for IBD prompt energy

(c) Fit result of far target for electron energy spectrum (d) Fit result of near target for electron energy spectrum 


\section{Energy Scale uncertainty.}

We could draw source data peak distribution adapting p.e to $\mathrm{MeV}$ conversion function. Energy scale differences by comparing far and near source peak are under $0.15 \%$ level. It means energy conversion function well converted from charge to $\mathrm{MeV}$ for each detectors as identical detectors.

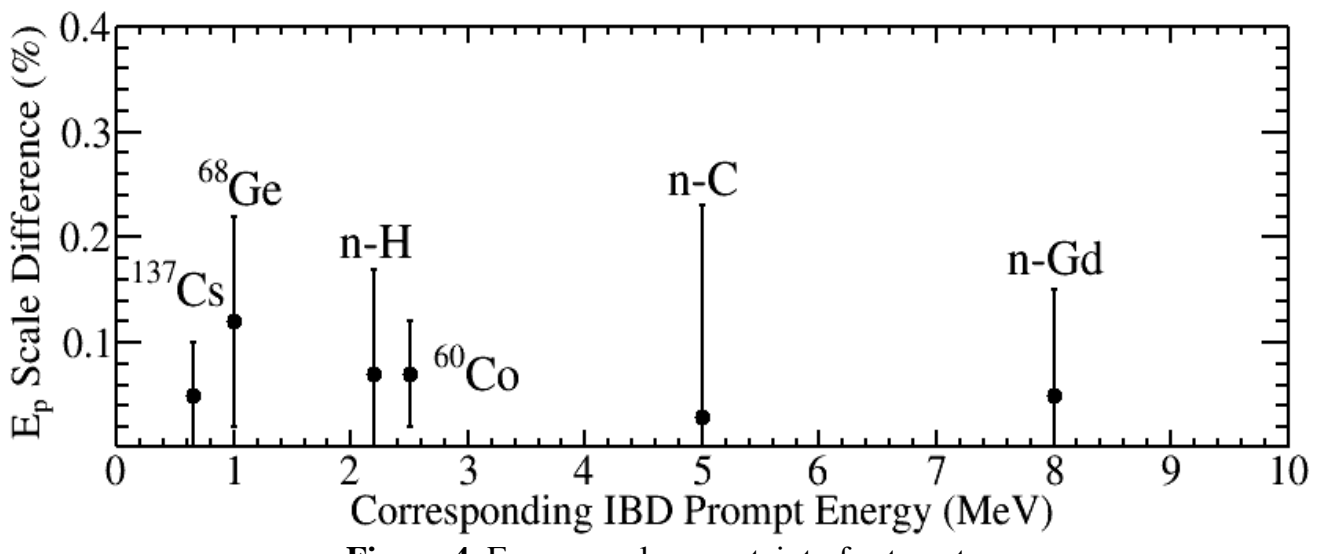

Figure 4. Energy scale uncertainty for target

\section{Summary.}

Non-linear response of scintillating energy for IBD prompt signal is well understood to obtain conversion functions. Energy scale difference between far and near is estimated 